Int. J. Morphol.,

23(4):363-368, 2005.

\title{
Inervación del Músculo Semimembranoso
}

\author{
Innervation of the Semimembronosus Muscle \\ Enrique Olave
}

OLAVE, E. Inervación del músculo semimembranoso. Int. J. Morphol., 23(4):363-368, 2005.

RESUMEN: La inervación de los músculos es descrita de forma general en la mayoría de los textos de Anatomía. La localización biométrica de sus ramos musculares y puntos motores son temas no totalmente esclarecidos, por lo que es necesario tener datos precisos sobre ellos, los que son de importancia cuando se trata de ubicarlos con propósitos de electroestimulación o de inyección de algunos agentes neurolíticos.

Se utilizaron 28 muestras de 14 cadáveres formolizados de individuos adultos, brasileños, de sexo masculino. A través de disección, se estudiaron los ramos que inervan al músculo semimembranoso. Para registros biométricos se consideraron como puntos de referencia, a la parte más distal de la tuberosidad isquiática (TI) y una línea transversal trazada entre las partes más salientes de los cóndilos femorales (LBE).

En 18 de los casos se observó 1 ramo de inervación para el músculo procedente del nervio isquiático, en los restantes 10 se presentaron 2 ramos de inervación. En 60,7 \% de los casos su ramo derivó de un tronco común con el del aductor magno. En el grupo con un ramo, la distancia promedio entre el origen de R1 respecto a la TI fue de $121,8 \mathrm{~mm} \pm 24,6$ en el lado derecho y de 124,7mm $\pm 15,9$ en el izquierdo. Cuando se presentaron 2 ramos de inervación, la distancia promedio para R1 en el lado derecho fue de $125,5 \mathrm{~mm} \pm 28$ y para R2 fue de $123,7 \mathrm{~mm} \pm 29,2$; en el lado izquierdo para $\mathrm{R} 1$ fue de $111,5 \mathrm{~mm} \pm 28,2$ y para $\mathrm{R} 2$ de $149,4 \mathrm{~mm} \pm 36,8$. Con respecto a la longitud de los ramos, cuando se presentó sólo 1 , el promedio en el lado derecho fue de $38,7 \mathrm{~mm} \pm 26,6$ y en el lado izquierdo de $28,3 \mathrm{~mm} \pm 23,1$. Los ramos principales se dividieron en 2 , 3 y más ramos secundarios (RS), de los cuales se registró la localización de los puntos motores correspondientes.

Estos resultados contribuirán a complementar el conocimiento de la inervación y de los puntos motores de la musculatura del muslo.

PALABRAS CLAVE: Anatomía; Inervación; Músculo semimembranoso; Puntos motores.

\section{INTRODUCCIÓN}

La inervación de los músculos es descrita de forma general en la mayoría de los textos de Anatomía (Testut \& Latarjet, 1969; Healey \& Seybold, 1972; Williams et al., 1995; Snell, 2002; Moore \& Dalley, 2003). La localización biométrica de sus ramos musculares y puntos motores son temas no totalmente esclarecidos, por lo que se hace necesario tener datos precisos sobre ellos, los que son de importancia cuando se trata de ubicar tales puntos con propósitos de electroestimulación o de inyección de algunas sustancias neurolíticas (Seidel et al., 1996; Liu et al., 1997). Con el objetivo de complementar el conocimiento de la inervación del músculo semimembranoso y localizar sus puntos motores, se realizó un estudio en que se registraron una serie de parámetros biométricos sobre sus nervios y divisiones secundarias tales como: origen, longitud y puntos de penetración motora respecto de un punto de referencia que puede ser localizado externamente sobre la piel, datos que pueden ser utilizados como base para diversos procedimientos quirúrgicos y de electroestimulación que se realizan en la zona posterior del muslo y especialmente, los relacionados con los nervios motores de la misma.

\section{MATERIAL Y MÉTODO}

Para la presente investigación se utilizaron 28 muestras pertenecientes a 14 cadáveres formolizados de individuos adultos, brasileros, todos de sexo masculino. El estudio de los ramos que inervan al músculo semimembranoso se realizó a través de disección, auxiliado por una lupa de 3 a 5 veces de aumento, en caso de los ramos musculares menores. Después de disecados los nervios respectivos, se consideraron como puntos de referencia, la parte más distal de la tuberosidad isquiática (TI) y una línea transversal trazada entre las partes más salientes de los cóndilos femorales (LBE), registrándose los siguientes parámetros: 
- Número de ramos que alcanzan al músculo semimembranoso (R1, R2).

- Distancia entre la TI y el origen del ramo muscular.

- Longitud de los ramos musculares.

- División del ramo muscular en ramos secundarios (RS).

- Distancia entre TI y los puntos de penetración en el vientre muscular (puntos motores).

- Localización de los puntos motores respecto a tercios proximal, medio o distal de la distancia TI-LBE.

Para el registro de las medidas mencionadas, se utilizó un caliper digital, de precisión 0,05 mm.

Todas las muestras fueron esquematizadas y descritas en detalle, fotografiando las más representativas. Los datos obtenidos fueron analizados con estadística descriptiva y comparación de muestras pareadas utilizando el programa SPSS 11.0.

\section{RESULTADOS}

De los 28 casos estudiados, en 18 de ellos se observó 1 ramo de inervación para el músculo en estudio procedente de la porción tibial del nervio isquiático, en los restantes 10 se presentaron 2 ramos de inervación. En ambos lados hubo 9 casos con 1 ramo.

Del total de muestras (28), en 17 casos $(60,7 \%)$ se observó un tronco común para los músculos semimembranoso y aductor magno. En los 11 casos restantes $(39,3 \%)$ se encontró un tronco para los músculos semitendinoso, aductor magno y semimembranoso (Fig. 1).

Las distancias promedio entre el origen de los ramos R1 y R2 respecto a la TI están expresadas en la Tabla I. Al comparar los lados, tanto en R1 como en R2 las diferencias no fueron estadísticamente significativas.

Considerando el total de muestras (28) y dividiendo la distancia TI-LBE en tercios, el origen de R1 ocurrió en 22 casos $(78,6 \%)$ a nivel del tercio medio de esta distancia, encontrándose tal disposición bilateralmente. De éstos, el R1 se originó de la parte proximal del tercio mencionado en 20 $(90,9 \%)$ y de su parte media en sólo 2 casos.
En las 6 muestras restantes $(21,4 \%)$, el R1 se originó en el tercio proximal de la distancia TI-LBE, específicamente de su parte distal.

Con respecto a la longitud de los ramos, cuando se presentó sólo uno (18 casos) el promedio fue de $33,5 \mathrm{~mm} \pm$ 24,7 . En el lado derecho fue de $38,7 \mathrm{~mm} \pm 26,6$ y en el lado izquierdo fue de $28,3 \mathrm{~mm} \pm 23,1$. Al comparar ambos lados, la diferencia no fue estadísticamente significativa.

Cuando se presentaron dos ramos, para el R1 de ambos lados se obtuvo un promedio de $27,1 \mathrm{~mm} \pm 14,7$ y para el R2 de 63,9mm $\pm 28,0$. En el lado derecho, el promedio de la longitud de $\mathrm{R} 1$ fue de $30,1 \mathrm{~mm} \pm 17,9$ y de R2 de $65,9 \mathrm{~mm}$ $\pm 30,8$, en el lado izquierdo para R1 se registró un promedio de $24,1 \mathrm{~mm} \pm 11,9$ y para $\mathrm{R} 2$ de $61,9 \mathrm{~mm} \pm 28,4$. Al comparar R1 y R2 de ambos lados, las diferencias no fueron estadísticamente significativas.

Con respecto a la división en ramos secundarios (RS), el número de RS derivados de los R1 y R2, cuando presente, en relación al número de casos están expresados en la Tabla II. (Fig.2)

Tabla II. Número de ramos secundarios (RS) derivados de la división de los ramos R1 y R2 en el músculo semimembranoso.

\begin{tabular}{lcccc}
\hline & \multicolumn{2}{c}{ Lado } & $\begin{array}{c}\text { derecho } \\
\text { Lado }\end{array}$ & $\begin{array}{c}\text { izquierdo } \\
\text { División }\end{array}$ \\
R1 & R2 & R1 & R2 \\
\hline Directo & 1 & - & 2 & 2 \\
En 2 RS & 4 & 2 & 7 & 3 \\
En 3 RS & 8 & 2 & 3 & - \\
En 4 RS & 1 & 1 & 1 & - \\
En 5 RS & - & - & - & - \\
En 6 RS & - & - & 1 & - \\
\hline Total de casos & 14 & 5 & 14 & 5 \\
\hline
\end{tabular}

Las distancias promedios entre el punto motor de los diferentes RS (RS1,RS2,y RS3) de R1, R2 con respecto a la TI, se muestran en las Tablas III y IV.

Asociando las distancias desde la TI al punto de ingreso en el vientre muscular de los RS1, RS2 y RS3, ramos de R1 se muestran en las taTablas V y VI.

Tabla III. Distancias promedio del punto de ingreso de los ramos secundarios (RS) de R1 respecto a la tuberosidad isquiática, en las muestras con un ramo de inervación para el músculo semimembranoso (en $\mathrm{mm}$ ).

\begin{tabular}{lccc}
\hline R1 & Lado derecho & Lado izquierdo & Ambos lados \\
\hline S1 & $163,1 \pm 37,4$ & $176,6 \pm 14,8$ & $169,8 \pm 28,5$ \\
S2 & $200,1 \pm 41,1$ & $221,9 \pm 32,9$ & $211,0 \pm 37,8$ \\
S3 & $218,4 \pm 21,0$ & $206,1 \pm 6,40$ & $213,9 \pm 17,70$ \\
\hline
\end{tabular}

Tabla I. Distancias promedio entre el origen de R1 y R2 respecto a la tuberosidad isquiática, en muestras con uno y dos ramos de inervación para el músculo semimembranoso (en mm).

\begin{tabular}{lllll}
\hline & \multicolumn{2}{c}{ Con 2 } & ramos & \multicolumn{2}{c}{ Con 1 } & ramo \\
& Lado derecho & Lado izquierdo & Lado derecho & Lado izquierdo \\
\hline R1 & $125,5 \pm 28,0$ & $111,5 \pm 28,2$ & $121,8 \pm 24,6$ & $124,7 \pm 15,9$ \\
R2 & $123,7 \pm 29,2$ & $149,4 \pm 36,8$ & & \\
\hline
\end{tabular}




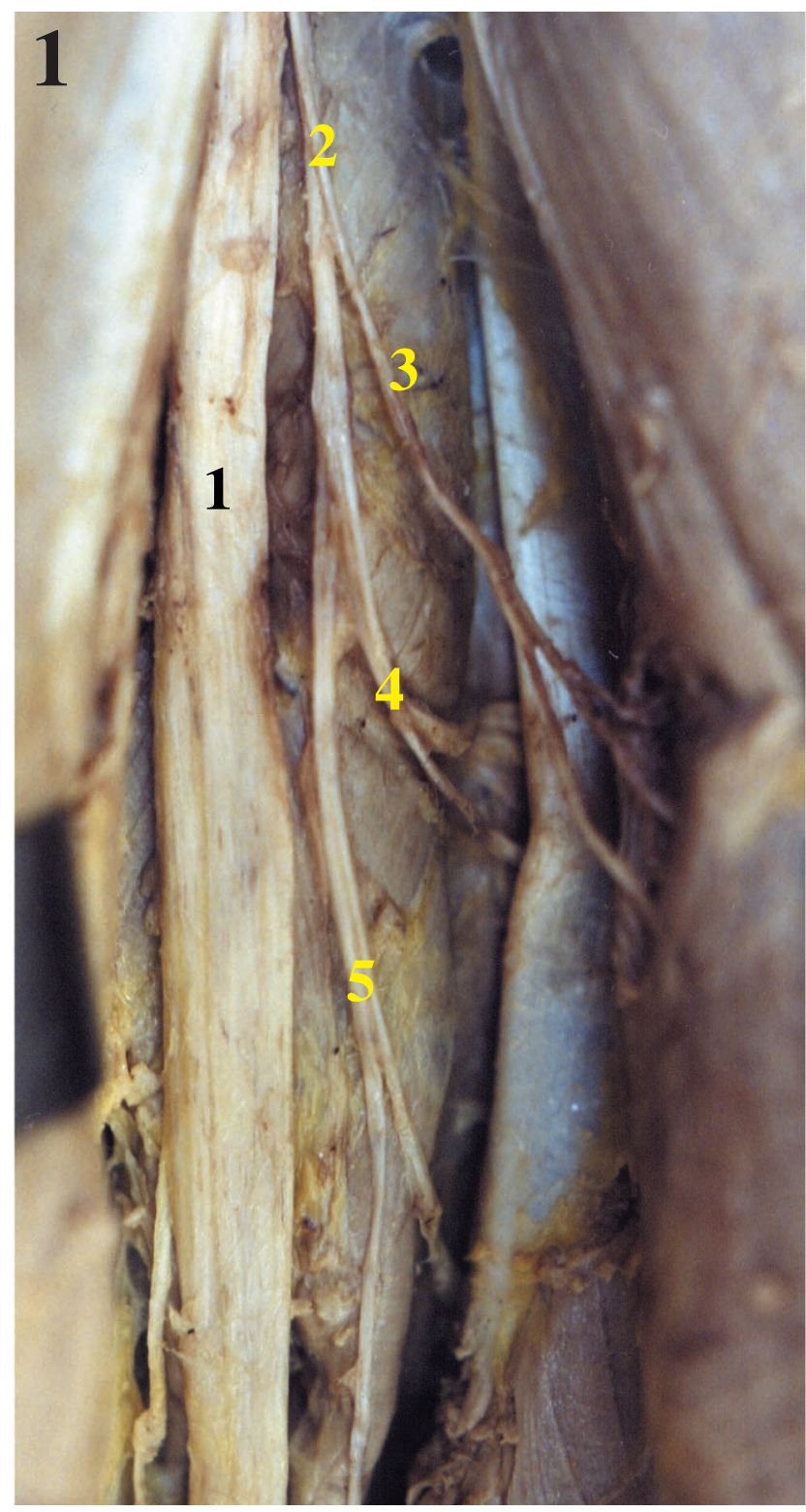

Fig. 1. 1. Nervio isquiático; 2. Tronco común para los mm. semitendinoso, aductor magno y semimembranoso; 3. Ramo para el m. semitendinoso; 4. Ramo para el m. aductor magno; 5 . Ramo para el m. semimembranoso.

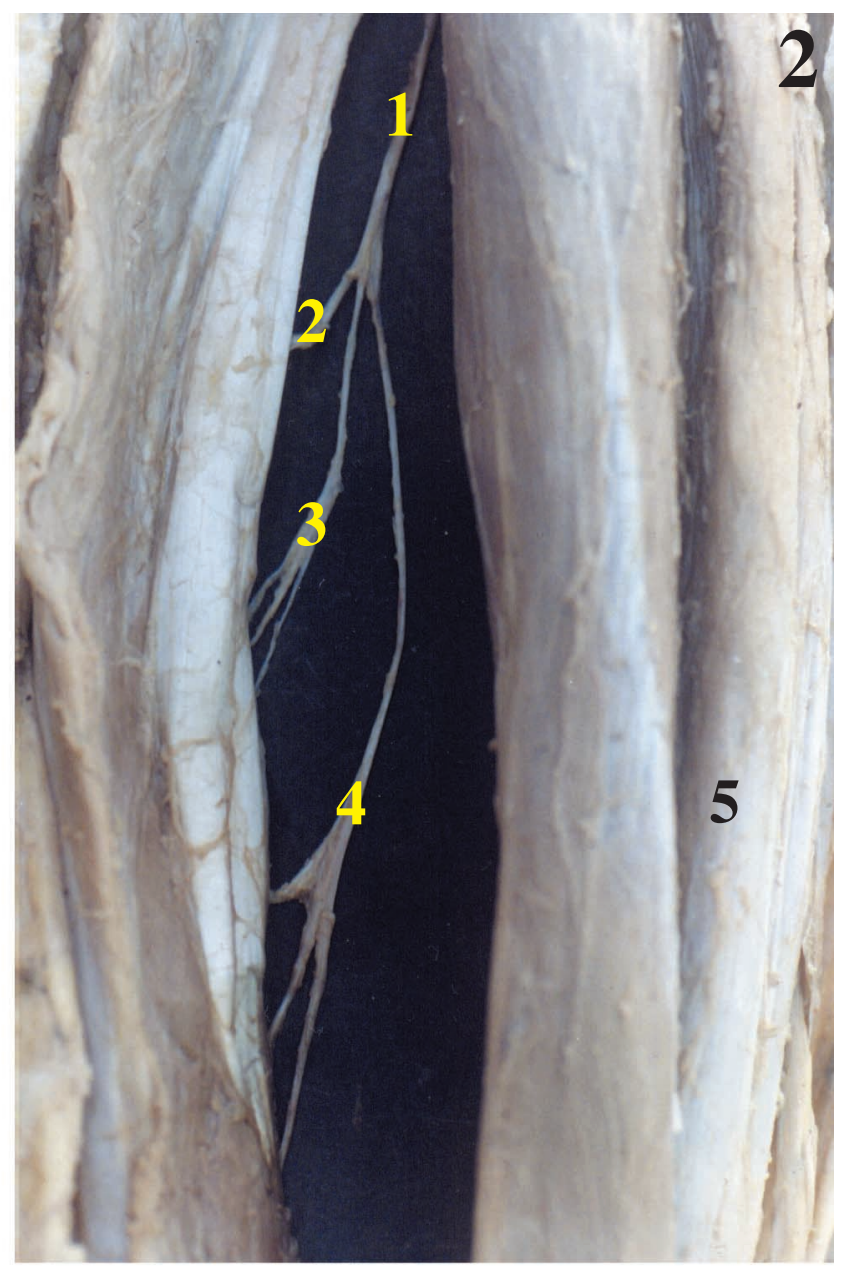

Fig. 2. 1. Ramo 1 para el m. semimembranoso (R1); 2. Ramo secundario 1 (RS1) para el m. semimembranoso; 3. Ramo secundario 2 (RS2) para el m. semimembranoso; 4. Ramo secundario 3 (RS3) para el m. semi-membranoso; 5. m. bíceps femoral.

Tabla V. Localización de los puntos de ingreso de los ramos secundarios de R1 en todas las muestras respecto a la división en tercios de la distancia TI-LBE

\begin{tabular}{lcccccc}
\hline Tercios & S1 & $\%$ & S2 & $\%$ & S3 & $\%$ \\
Proximal & - & - & - & - & - & - \\
Medio & 28 & 100 & 19 & 76 & 12 & 85,7 \\
Distal & - & - & 6 & 24 & 2 & 14,3 \\
Total & 28 & 100 & 25 & 100 & 14 & 100,0 \\
\hline
\end{tabular}

Tabla IV. Distancias promedio del punto de ingreso de los ramos secundarios (RS) de R1 y R2 respecto a la tuberosidad isquiática, en las muestras con dos ramos de inervación para el músculo semimembranoso (en mm).

\begin{tabular}{lllll}
\hline & & R1 & R2 \\
S1 & Lado derecho & Lado izquierdo & Lado derecho & Lado izquierdo \\
S2 & $151,5+28,7$ & $140,5+16,0$ & $195,6+22,9$ & $213,0+31,9$ \\
\hline
\end{tabular}

Tabla VI. Localización de los puntos de ingreso de los ramos secundarios de R1 en todas las muestras dentro del tercio medio.

\begin{tabular}{lrrrrrr}
\hline Tercio Medio & S1 & $\%$ & S2 & $\%$ & S3 & $\%$ \\
Parte Proximal & 7 & 25,0 & 2 & 10,5 & - & - \\
Parte Media & 19 & 67,9 & 12 & 63,2 & 2 & 16,7 \\
Parte Distal & 2 & 7,1 & 5 & 26,3 & 10 & 83,3 \\
Total de casos & 28 & 100,0 & 19 & 100,0 & 12 & 100,0 \\
\hline
\end{tabular}


Los puntos de ingreso de los RS de R2 respecto a los tercios de la distancia TI-LBE, se muestran en la Tabla VII.

Tabla VII. Localización de los puntos de ingreso de los ramos secundarios de R2 en todas las muestras respecto a la división en tercios de la distancia TI-LBE.

\begin{tabular}{lcccccc}
\hline Tercios & S1 & $\%$ & S2 & $\%$ & S3 & $\%$ \\
\hline Proximal & - & - & - & - & - & - \\
Medio & 7 & 70 & 3 & 37,5 & 1 & 33,3 \\
Distal & 3 & 30 & 5 & 62,5 & 2 & 66,7 \\
Total & 10 & 100 & 8 & 100 & 3 & 100 \\
\hline
\end{tabular}

\section{DISCUSIÓN}

El músculo semimembranoso se localiza en la región posterior del muslo, originándose en la parte superolateral de la tuberosidad isquiática y por una expansión lateral en la parte adyacente de la rama del isquion. Sus fibras se encuentran parcialmente entrelazadas con aquellas de los músculos bíceps femoral y del semitendinoso. Este complejo tendón proximal se expande en una aponeurosis, descendiendo anterior a los mm. semitendinoso y bíceps femoral. A partir de ésta, las fibras musculares convergen hacia una segunda aponeurosis sobre la cara posterior distal del músculo que se afina en su tendón de fijación distal, el cual se divide a nivel de la rodilla, en 5 componentes, siendo el principal aquel que se fija en la cara posterior del cóndilo medial de la tibia. Se encuentra inervado por la porción tibial del nervio isquiático (Williams et al.).

La información sobre la de inervación de los diferentes músculos del cuerpo se encuentra en los clásicos textos de Anatomía (Testut \& Latarjet; Healey \& Seybold; Williams et al.; Snell; Moore \& Dalley), siendo adecuada para los procedimientos quirúrgicos de rutina, pero aún, faltan detalles suficientes para servir de soporte anatómico a reconstrucciones de complejas lesiones y situaciones de parálisis de nervios periféricos. Sin embargo, diversos autores (Sunderland; Buchanan \& Eriksson, 1996; Seidel, et al.; Rab et al., 1997; Olave et al., 2002; Olave \& Braga, 2003 y Olave, 2004) han hecho interesantes aportes al conocimiento de detalles anatómicos y biométricos que han ido más allá de la simple descripción del ramo principal de inervación.

El conocimiento de la posición de los ramos nerviosos y sus terminaciones en el vientre muscular facilitan la inserción de electrodos en los puntos motores de éstos para estimulaciones eléctricas adecuadas en determinadas lesiones (Liu et al.). Además, la posición de éstos permite realizar estudios electromiográficos así como también bloqueos nerviosos motores selectivos.
En relación al músculo en estudio, Sunderland señaló que el nervio para el músculo semimembranoso era común con el del músculo aductor magno, y, que este tronco común se desprendía de la porción tibial del nervio isquiático, dentro de $\operatorname{los} 10 \mathrm{~cm}$ siguientes a la tuberosidad isquiática. Si bien es cierto, esta descripción corresponde a lo observado en este estudio, el nervio común se originó en la mayoría de los casos, sobre la distancia mencionada por este autor, la que calculamos en promedio, considerando ambos lados en $123 \mathrm{~mm}$, o sea, $20 \mathrm{~mm}$ más que sus observaciones. Este último resultado es más concordante con Seidel et al., que registraron una medida para el nervio principal del semimembranoso de $131 \mathrm{~mm} \pm 36$.

Por otra parte, este mismo autor señaló que encontró en 14 de 20 muestras (70\%), un tronco común para la inervación de los músculos semitendinoso ( $2^{\circ}$ ramo), semimembranoso y aductor magno. Considerando los resultados de este estudio, el mencionado por Sunderland está muy por encima de nuestras observaciones, ya que sólo encontramos esta situación en 11 casos de los 28 (39,3\%) estudiados. En las restantes muestras, la inervación del músculo en estudio proviene de un tronco común que inerva también al músculo aductor magno, el que recibe inervación tanto del nervio isquiático como del nervio obturador, concordando con Healey \& Seybold. Por su parte, Goss (1977) describió que el músculo semimembranoso recibe varios ramos de la porción tibial del nervio isquiático, sin indicar número específico ni alusión a tronco común con otros nervios musculares, lo cual no parece adecuado ya que sólo encontramos uno o dos ramos principales inervando a este músculo y tal vez, se refiera a los ramos secundarios que sí, son varios.

Recientemente, Woodley \& Mercer (2005) estudiaron su arquitectura, identificando tres regiones dentro del músculo. Sin embargo, ésta no fue congruente con los padrones de inervación, ya que, un primer ramo inervó sólo dos regiones y la tercera recibió un ramo secundario. 
La presente investigación reveló que el músculo semimembranoso recibe, en más del $60 \%$ de los casos, sólo un ramo de inervación y dos ramos en el porcentaje restante. Sin embargo, al observar los resultados biométricos se puede determinar que los nervios se originan y penetran a nivel del tercio medio de la distancia TI-LBE.

En general, la literatura consultada no revela detalles biométricos con respecto a los nervios del músculo semimembranoso, por lo que, los aquí informados servirán de referencia para diversos procedimientos. Relacionando la biometría realizada con la anatomía de superficie, se puede señalar que el origen del ramo dirigido a este músculo se localiza en la parte posterior del muslo, a nivel de la línea media, en alrededor del $80 \%$ de los casos en el tercio medio de la distancia TI-LBE y de este porcentaje, en más del 90 $\%$ lo hace a nivel de la parte proximal del tercio aludido. En el porcentaje restante, su origen se localizó a nivel del tercio proximal de la distancia TI-LBE.

Con relación a los puntos motores, se puede indicar que en la totalidad de los casos, el punto de ingreso del RS1 de R1 fue a nivel del tercio medio de la distancia TI-LBE y en su mayoría a nivel de la parte media de este tercio. Por su parte, el RS2 en la mayor parte de los casos (19/25) ingresó en el vientre muscular a nivel del tercio medio, y al igual que el precedente, un gran porcentaje a nivel de su parte media. En general, tanto el nivel de origen como los puntos motores de los primeros dos ramos de división del nervio principal, se localizan a nivel del tercio medio de la distancia TI-LBE, resaltando que su origen es a nivel de la parte proximal y los puntos motores a nivel de la parte media del tercio aludido.

De los 14 casos en que se presentó el nervio principal dividido en 3 ramos secundarios, el RS3, también penetró en el vientre muscular a nivel del tercio medio en más del $80 \%$ de los casos y haciéndolo en la parte distal de éste.

Cuando se presenta un R2, éste se divide con más frecuencia en RS1 y RS2, y en pocas ocasiones se observa un RS3. En la mayoría de los casos, el primer ramo secundario penetra en el vientre muscular a nivel del tercio medio, en cambio el segundo ramo lo hace un poco más distante de la TI, ya que se localiza en el tercio distal un mayor número de veces, aunque en su parte proximal. Ya el RS3, cuando presente, ingresa principalmente en el tercio distal.

De lo mencionado anteriormente, se puede concluir que la inervación del músculo semimembranoso, generalmente se origina y es recibida dentro del tercio medio de la distancia TI-LBE. Sin duda, estos datos biométricos aplicados a la zona posterior del muslo y tomando como puntos de referencia a la tuberosidad isquiática y a las partes más salientes de los cóndilos del fémur, permitirán una localización más o menos exacta del nivel de origen y de los puntos motores del músculo en cuestión. Así, procedimientos que necesiten de la colocación de electrodos en éstos, para tratamientos de estimulación o inyección de sustancias neurolíticas, podrán basarse en los datos aportados por esta investigación.

OLAVE, E. Innervation of the semimembranosus muscle. Int. J. Morphol., 23(4):363-368, 2005.

SUMMARY: Classical Anatomy books describe muscle innervation in a very general way. As an example biometric location of muscular branches and their respective motor points are not quite clear. Therefore, it is necessary to know their exact position when therapies such as electric stimulation or injection of neurolytic substances are applied. On this purpose to shed some light on these matters, it was studied the semimembranosus muscle innervation on 28 lower limbs specimens of 14 formalyzed cadavers from adult male of Brazilian origin.

In order to obtain their biometric records, the landmark points employed here were arranged from the distal part of the ischiatic tuberosity (IT) followed by an imaginary transversal line deployed over the most prominent parts of the femoral epicondyles.

On 18 cases it was observed only one innervation branch, coming from the tibial portion of the ischiatic nerve directed to the semimembranosus muscle and in the remaining 10 cases it was observed that two branches were received by the semimembranosus muscle. In $60,7 \%$ of cases the branch originated from a common trunk together at the magnus adductor muscle branch. In the group with one branch, the average distance from the R1 origin respect to IT was $121,8 \mathrm{~mm} \pm 24,6$ at the right side and 124,7 $\mathrm{mm} \pm 15,9$ at the left side.

In the group with two branches the average distance at the right side for both $\mathrm{R} 1$ was $125,5 \mathrm{~mm}+28,0$ and for $\mathrm{R} 2123,7 \mathrm{~mm} \pm 29,2$. At the left side both distances were $111,5 \mathrm{~mm} \pm 28,2$ for $\mathrm{R} 1$ and $149,4 \mathrm{~mm} \pm 36,8$ for $\mathrm{R} 2$. In the one branch group at the right side the R1average length gave a value of $38,7 \mathrm{~mm} \pm 26,6$ instead for the left side gave a value of $28,3 \mathrm{~mm} \pm 23,1$. The R1 and R2 were distributed in 2,3 and more secondary branches regarding the motor points location.

Finally, I hope with these findings to contribute at the innervation knowledge and the motor point areas from thigh muscles.

KEY WORDS: 1. Anatomy, 2. Innervation, 3. Semimembranosus muscle, 4. Motor points. 


\section{REFERENCIAS BIBLIOGRÁFICAS}

Buchanan, T. S. \& Eriksson, J. Selective block of the brachialis motor points. An anatomic investigation of musculocutaneous nerve branching. Reg. Anesth., 21(2):89-92, 1996.

Goss, Ch. M. Gray Anatomía. Barcelona, Salvat, 1977.

Healey, J. \& Seybold, W. Anatomía Clínica. $1^{\mathrm{a}}$ ed. México, Interamericana, 1972. $324 \mathrm{p}$.

Liu, J; Pho. R.W.; Pereira, B. P.; Lau, H. K. \& Kumar, V. P. Distribution of primary motor nerve branches and terminal nerve entry points to the forearm muscles. Anat. Rec., 248(3):456-63, 1997.

Moore, K. \& Dalley, Anatomía con orientación clínica. Buenos Aires, Panamericana, 2003.

Olave, E. \& Braga, M. T. T. Inervación del músculo semitendinoso, biometría de sus ramos y localización de sus puntos motores. Int. J. Morphol., 21(2):161-5, 2003.

Olave, E.: Gabrielli, C.; Braga, M. T. T. \& del Sol, M. Aspectos biométricos de los ramos motores del nervio musculocutáneo para el músculo braquial. Rev. Chil. Anat., 20(2):231-6, 2002.

Olave, E. Aspectos anatómicos y biométricos de la inervación del músculo coracobraquial y sus puntos motores. Int. J. Morphol., 22(4):323-6, 2004.

Rab, M.; Mader, N.; Kamolz, L. P.;Hausner, T.; Gruber, H. $\&$ Girsch, W. Basic anatomical investigation of semitendinosus and the long head of bíceps femoris muscle for their posible use in electrically stimulated neosphincter formation. Surg. Radiol. Anat., 19:287-291, 1997

Seidel, P. M.: Seidel, G. K.; Gans, B. M. \& Dijkers, M. Precise localization of the motor nerve branches to the hamstring muscles: an aid to the conduct of neurolytic procedures. Arch. Phys. Med. Rehábil., 77(11):1157-60, 1996.

Snell, R. Anatomia Clinica para Estudantes de Medicina. $5^{\mathrm{a}}$ ed. Rio de Janeiro, Guanabara Koogan, 2002.

Sunderland, S. Nervios periféricos y sus lesiones. Barcelona, Salvat, 1985. 1054 p.
Testut, L. \& Latarjet, M. Tratado de Anatomía Humana. Barcelona, Salvat, 1969.

Williams, P.; Warwick, R.; Dyson, M. \& Bannister, L. Gray Anatomía. 37 ed. Rio de Janeiro, Guanabara Koogan, 1995.

Woodley, S. J. \& Mercer, S. R. Hamstring muscles: architecture and innervation. Cells tissues organs, 179(3):125-41, 2005.

Dirección para correspondencia: Prof. Dr. Erique Olave R.

Facultad de Medicina

Universidad de La Frontera

Casilla $54-\mathcal{D}$

Temuco - CHILE

Email:eolave@ufro.cl

Recibido : 10-06-2005

Aceptado: 12-09-2005 